\title{
Almost Automorphic Solutions for Fuzzy Cohen-Grossberg Neural Networks with Mixed Time Delays
}

\author{
Yongkun Li, ${ }^{1}$ Xiaofang Meng, ${ }^{1}$ and Xuemei Zhang ${ }^{2}$ \\ ${ }^{1}$ Department of Mathematics, Yunnan University, Kunming, Yunnan 650091, China \\ ${ }^{2}$ School of Mathamatics and Information Science, Qujing Normal University, Qujing, Yunnan 655011, China \\ Correspondence should be addressed to Yongkun Li; yklie@ynu.edu.cn
}

Received 7 December 2014; Accepted 17 February 2015

Academic Editor: Sotiris K. Ntouyas

Copyright (c) 2015 Yongkun Li et al. This is an open access article distributed under the Creative Commons Attribution License, which permits unrestricted use, distribution, and reproduction in any medium, provided the original work is properly cited.

\begin{abstract}
This paper is concerned with the problem of almost automorphic solutions of a class of fuzzy Cohen-Grossberg neural networks with mixed time delays and variable coefficients. Based on inequality analysis techniques and combining the exponential dichotomy with fixed point theorem, some sufficient conditions for the existence and global exponential stability of almost automorphic solutions are obtained. Finally, a numerical example is given to show the feasibility of our results.
\end{abstract}

\section{Introduction}

In recent years, neural networks have been extensively studied due to their important applications in many areas such as function approximation, pattern recognition, associative memory, and combinatorial optimization. In particular, the research on the dynamical behavior of the neural networks has become an important topic in neural network theory. Therefore, the dynamics of neural networks, especially the existence of periodic solutions, antiperiodic solutions, and almost periodic solutions to neural networks, has been extensively investigated and a large number of criteria on the stability of neural networks have been discussed in the literature [1-5]. Moreover, it is well known that CohenGrossberg neural network [6] is one of the most popular and typical network models. Some models such as Hopfield-type neural networks, CNNs, BAM-type models are special cases of Cohen-Grossberg neural networks. For more details about Cohen-Grossberg neural networks, one can see, for example, [7-12].

In reality, time delays often occur due to finite switching speeds of the amplifiers and communication time. Moreover, it is observed both experimentally and numerically that time delay in neural networks may induce instability. Therefore, neural networks with time delays have recently become a topic of research interest. On the other hand, in mathematical modeling of real world problems, we encounter some inconveniences besides delays, namely, the complexity and the uncertainty or vagueness. Vagueness is opposite to exactness and we argue that it cannot be avoided in the human way of regarding the world. Any attempt to explain an extensive detailed description necessarily leads to using vague concepts since precise description contains abundant number of details. To understand it, we must group them together and this can hardly be done precisely. A nonsubstitutable role is here played by natural language. For the sake of taking vagueness into consideration, fuzzy theory is viewed as a more suitable setting. Based on traditional CNNs, T. Yang and L.-B. Yang [13] first introduced the fuzzy cellular neural networks (FCNNs), which integrate fuzzy logic into the structure of traditional CNNs and maintain local connectedness among cells. Unlike previous CNNs, FCNN is a very useful paradigm for image processing problems, which has fuzzy logic between its template input and/or output besides the sum of product operation. It is a cornerstone in image processing and pattern recognition. Recently, some results on stability and other behaviors have been derived for fuzzy neural networks with or without time delays (see [1422]).

Also, the almost periodicity is more universal than the periodicity. Moreover, almost automorphic functions, which were introduced by Bochner in his papers [23-25] in 
relation to some aspects of differential geometry, are much more general than almost periodic functions. The notion of almost automorphic function was introduced to avoid some assumptions of uniform convergence that arise when using almost periodic function. In the last several decades, the basic theories on the almost automorphic functions have been well developed $[26,27]$ and have been applied successfully to the investigation of almost automorphic solutions of differential equations (see [28-30] and reference therein). In [31-33], authors studied almost automorphic solutions for several classes of neural networks, respectively. Recently, considerable efforts have been devoted to the periodic solutions and almost periodic solutions of Cohen-Grossberg neural networks with time delays (see, e.g., [34-41]). However, to the best of our knowledge, there is no paper published on the existence and stability of almost automorphic solutions for fuzzy neural networks with time delays.

Motivated by the above discussion, in this paper, we propose a class of fuzzy Cohen-Grossberg neural networks with mixed time delays and variable coefficients as follows:

$$
\begin{aligned}
x_{i}^{\prime}(t)=- & a_{i}\left(x_{i}(t)\right) \\
& \cdot\left[b_{i}\left(x_{i}(t)\right)-\sum_{j=1}^{n} c_{i j}(t) f_{j}\left(x_{j}(t)\right)\right. \\
& -\sum_{j=1}^{n} d_{i j}(t) g_{j}\left(x_{j}\left(t-\tau_{i j}(t)\right)\right) \\
& -\sum_{j=1}^{n} e_{i j}(t) \mu_{j}(t)-\bigwedge_{j=1}^{n} \alpha_{i j}(t) \\
& \cdot \int_{-\infty}^{t} K_{i j}(t-s) h_{j}\left(x_{j}(s)\right) d s \\
& -\bigwedge_{j=1}^{n} T_{i j}(t) \mu_{j}(t) \\
& -\bigvee_{j=1}^{n} \beta_{i j}(t) \int_{-\infty}^{t} K_{i j}(t-s) h_{j}\left(x_{j}(s)\right) d s \\
& \left.-\bigvee_{j=1}^{n} S_{i j}(t) \mu_{j}(t)-I_{i}(t)\right],
\end{aligned}
$$

where $i=1,2, \ldots, n, n$ corresponds to the number of units in neural networks; $x_{i}(t)$ corresponds to the state of the $i$ th unit at time $t ; a_{i}\left(x_{i}(t)\right)$ represents an amplification function at time $t ; b_{i}\left(x_{i}(t)\right)$ is an appropriately behaved function at time $t$ such that the solutions of model (1) remain bounded; $\alpha_{i j}$, $\beta_{i j}$, $T_{i j}$, and $S_{i j}$ are the elements of fuzzy feedback MIN template, fuzzy feedback MAX template, fuzzy feed forward MIN template, and fuzzy feed forward MAX template, respectively; $d_{i j}$ weight the strength of the $i$ th neuron at the time $t$, $c_{i j}$ and $e_{i j}$ are the elements of feedback template and feed forward template; $\wedge, \vee$ denote the fuzzy AND and fuzzy OR operation, respectively; $f_{j}, g_{j}$, and $h_{j}$ are the activation functions; $\tau_{i j}(t) \geq 0$ is the time-varying delay caused during the switching and transmission processes; $K_{i j}$ is the delay kernel function; $I_{i}(t)$ denotes external input to the $i$ th neuron at time $t$.

The initial value of (1) is the following:

$$
x_{i}(s)=\varphi_{i}(s), \quad s \in(-\infty, 0],
$$

where $\varphi_{i} \in C((-\infty, 0], \mathbb{R}), i=1,2, \ldots, n$.

Our main purpose of this paper is by utilizing inequality analysis techniques and combining the exponential dichotomy with fixed point theorem, to study the existence and global exponential stability of almost automorphic solutions of (1). Our results of this paper are completely new.

Throughout this paper, we assume that the following conditions hold.

$\left(H_{1}\right)$ The amplification functions $a_{i}(\cdot)(i=1,2, \ldots, n)$ are continuously bounded; that is, there exist positive constants $a_{i}^{-}$and $a_{i}^{+}$such that

$$
0<a_{i}^{-} \leq a_{i}(x) \leq a_{i}^{+}, \quad \forall x \in \mathbb{R}, i=1,2, \ldots, n .
$$

$\left(H_{2}\right)$ The behaved functions $b_{i}(\cdot)(i=1,2, \ldots, n)$ are continuous with $b_{i}(0)=0$ and there exist constants $\lambda_{i}$ such that

$$
\begin{gathered}
\frac{b_{i}(u)-b_{i}(v)}{u-v} \geq \lambda_{i}>0, \\
\forall u, v \in \mathbb{R}, \quad u \neq v, \quad i=1,2, \ldots, n .
\end{gathered}
$$

$\left(H_{3}\right) f_{j}, g_{j}, h_{j} \in C(\mathbb{R}, \mathbb{R})$ are Lipschitcz continuous with positive Lipschitcz constants $L_{j}^{f}, L_{j}^{g}$, and $L_{j}^{h}$ such that, for all $u, v \in \mathbb{R}$,

$$
\begin{aligned}
& \left|f_{j}(u)-f_{j}(v)\right| \leq L_{j}^{f}|u-v|, \\
& \left|g_{j}(u)-g_{j}(v)\right| \leq L_{j}^{g}|u-v|, \\
& \left|h_{j}(u)-h_{j}(v)\right| \leq L_{j}^{h}|u-v| ;
\end{aligned}
$$

moreover, we supposed that $f_{j}(0)=g_{j}(0)=h_{j}(0)=$ $0, i=1,2, \ldots, n$.

$\left(H_{4}\right) c_{i j}(t), d_{i j}(t), e_{i j}(t), \alpha_{i j}(t), \beta_{i j}(t), T_{i j}(t), S_{i j}(t), I_{i}(t)$, and $\tau_{i j}(t)$ are all continuous almost automorphic functions defined on $\mathbb{R}$.

$\left(H_{5}\right)$ The kernel $K_{i j}(\cdot)$ is almost automorphic and it satisfies that

$$
\begin{array}{r}
\int_{0}^{+\infty} K_{i j}(s) d s=1, \quad \int_{0}^{+\infty} e^{\mu s} K_{i j}(s) d s<\infty \\
i, j=1,2, \ldots, n,
\end{array}
$$

where $\mu$ is a real positive number and there exist $\rho>0$ and $C>0$ such that $K_{i j}(t) \leq \rho e^{-t C}, i, j=1,2, \ldots, n$. 
For convenience, we introduce the following notations:

$$
\begin{aligned}
& c_{i j}^{+}=\sup _{t \in \mathbb{R}}\left|c_{i j}(t)\right|, \quad c_{i j}^{-}=\inf _{t \in \mathbb{R}}\left|c_{i j}(t)\right|, \\
& d_{i j}^{+}=\sup _{t \in \mathbb{R}}\left|d_{i j}(t)\right|, \quad d_{i j}^{-}=\inf _{t \in \mathbb{R}}\left|d_{i j}(t)\right|, \\
& e_{i j}^{+}=\sup _{t \in \mathbb{R}}\left|e_{i j}(t)\right|, \quad e_{i j}^{-}=\inf _{t \in \mathbb{R}}\left|e_{i j}(t)\right|, \\
& \alpha_{i j}^{+}=\sup _{t \in \mathbb{R}}\left|\alpha_{i j}(t)\right|, \quad \alpha_{i j}^{-}=\inf _{t \in \mathbb{R}}\left|\alpha_{i j}(t)\right|, \\
& \beta_{i j}^{+}=\sup _{t \in \mathbb{R}}\left|\beta_{i j}(t)\right|, \quad \beta_{i j}^{-}=\inf _{t \in \mathbb{R}}\left|\beta_{i j}(t)\right|, \\
& T_{i j}^{+}=\sup _{t \in \mathbb{R}}\left|T_{i j}(t)\right|, \quad T_{i j}^{-}=\inf _{t \in \mathbb{R}}\left|T_{i j}(t)\right|, \\
& S_{i j}^{+}=\sup _{t \in \mathbb{R}}\left|S_{i j}(t)\right|, \quad S_{i j}^{-}=\inf _{t \in \mathbb{R}}\left|S_{i j}(t)\right|, \\
& \tau=\max _{1 \leq i, j \leq n} \sup _{t \in \mathbb{R}}\left|\tau_{i j}(t)\right|, \\
& J_{i}(t)=\sum_{j=1}^{n} e_{i j}(t) \mu_{j}(t)+\bigwedge_{j=1}^{n} T_{i j}(t) \mu_{j}(t) \\
& +\bigvee_{j=1}^{n} S_{i j}(t) \mu_{j}(t)+I_{i}(t), \\
& J_{i}^{+}=\sup _{t \in \mathbb{R}}\left|J_{i}(t)\right|, \\
& J=\max _{1 \leq i \leq n}\left\{\omega_{i}^{-1} \frac{J_{i}^{+}}{\lambda_{i} a_{i}^{-}}\right\} \text {, }
\end{aligned}
$$

where $\omega_{i}>0, i, j=1,2, \ldots, n$.

This paper is organized as follows. In Section 2, we introduce some definitions and make some preparations for later sections. In Section 3, we present some sufficient conditions for the existence and global exponential stability of almost automorphic solutions of (1). In Section 4, we give an example to demonstrate the feasibility of our results.

\section{Preliminary}

In this section, we introduce some definitions and state some preliminary results.

Definition 1 (see [27]). A continuous function $f: \mathbb{R} \rightarrow \mathbb{R}^{n}$ is said to be almost automorphic if for every sequence of real numbers $\left(s_{n}^{\prime}\right)_{n \in \mathbb{N}}$ there exists a subsequence $\left(s_{n}\right)_{n \in \mathbb{N}}$ such that

$$
\lim _{n \rightarrow \infty} f\left(t+s_{n}\right)=g(t)
$$

is well defined for each $t \in \mathbb{R}$ and

$$
\lim _{n \rightarrow \infty} g\left(t-s_{n}\right)=f(t)
$$

for each $t \in \mathbb{R}$.
Remark 2. Note that the function $g$ in the definition above is measurable but not necessarily continuous. Moreover, if $g$ is continuous, then $f$ is uniformly continuous. Besides, if the convergence above is uniform in $t \in \mathbb{R}$, then $f$ is almost periodic. Denote by $A A\left(\mathbb{R}, \mathbb{R}^{n}\right)$ the collection of all almost automorphic functions

$$
A P\left(\mathbb{R}, \mathbb{R}^{n}\right) \subset A A\left(\mathbb{R}, \mathbb{R}^{n}\right) \subset B C\left(\mathbb{R}, \mathbb{R}^{n}\right),
$$

where $A P\left(\mathbb{R}, \mathbb{R}^{n}\right)$ and $B C\left(\mathbb{R}, \mathbb{R}^{n}\right)$ are the collection of all almost periodic functions and the set of bounded continuous functions from $\mathbb{R}$ to $\mathbb{R}^{n}$, respectively.

Lemma 3 (see [27]). Let $f, g \in A A\left(\mathbb{R}, \mathbb{R}^{n}\right)$. Then we have the following:

(i) $f+g \in A A\left(\mathbb{R}, \mathbb{R}^{n}\right)$;

(ii) $\lambda f \in A A\left(\mathbb{R}, \mathbb{R}^{n}\right)$ for any scalar $\lambda \in \mathbb{R}$;

(iii) $f_{\alpha} \in A A\left(\mathbb{R}, \mathbb{R}^{n}\right)$ where $f_{\alpha}: \mathbb{R} \rightarrow \mathbb{R}^{n}$ is defined by $f_{\alpha}(\cdot)=f(\cdot+\alpha)$

(iv) let $f \in A A\left(\mathbb{R}, \mathbb{R}^{n}\right)$; then the range $\mathbb{R}_{f}:=\{f(t), t \in \mathbb{R}\}$ is relatively compact in $\mathbb{R}^{n}$, thus $f$ is bounded in norm;

(v) if $\varphi: \mathbb{R}^{n} \rightarrow \mathbb{R}^{n}$ is a continuous function, then the composite function $\varphi \circ f: \mathbb{R}^{n} \rightarrow \mathbb{R}^{n}$ is almost automorphic;

(vi) $\left(A A\left(\mathbb{R}, \mathbb{R}^{n}\right),\|\cdot\|_{\infty}\right)$ is a Banach space.

Definition 4 (see [27]). A function $f \in C\left(\mathbb{R} \times \mathbb{R}^{n}, \mathbb{R}^{n}\right)$ is said to be almost automorphic in $t \in \mathbb{R}$ for each $x \in \mathbb{R}^{n}$ if for every sequence of real numbers $\left(s_{n}^{\prime}\right)_{n \in \mathbb{N}}$ there exists a subsequence $\left(s_{n}\right)_{n \in \mathbb{N}}$ such that

$$
\lim _{n \rightarrow \infty} f\left(t+s_{n}, x\right)=g(t, x)
$$

is well defined for each $t \in \mathbb{R}, x \in \mathbb{R}^{n}$ and

$$
\lim _{n \rightarrow \infty} g\left(t-s_{n}, x\right)=f(t, x)
$$

for each $t \in \mathbb{R}, x \in \mathbb{R}^{n}$. The collection of such functions will be denoted by $A A\left(\mathbb{R} \times \mathbb{R}^{n}, \mathbb{R}^{n}\right)$.

Lemma 5 (see [27]). Let $f: \mathbb{R} \times \mathbb{R}^{n} \rightarrow \mathbb{R}^{n}$ be an almost automorphic function in $t \in \mathbb{R}$ for each $x \in \mathbb{R}^{n}$ and assume that $f$ satisfies a Lipschitz condition in $x$ uniformly in $t \in \mathbb{R}$. Let $\varphi: \mathbb{R} \rightarrow \mathbb{R}^{n}$ be an almost automorphic function. Then the function

$$
\phi: t \longmapsto \phi(t)=f(t, \varphi(t))
$$

is almost automorphic.

Definition 6 (see [27]). System

$$
x^{\prime}(t)=A(t) x(t)
$$

is said to admit an exponential dichotomy if there exist a projection $P$ and positive constants $\alpha, \beta$ so that the fundamental solution matrix $X(t)$ satisfies

$$
\begin{gathered}
\left|X(t) P X^{-1}(s)\right| \leq \beta e^{-\alpha(t-s)}, \quad t \geq s, \\
\left|X(t)(I-P) X^{-1}(s)\right| \leq \beta e^{-\alpha(s-t)}, \quad t \leq s .
\end{gathered}
$$


Consider the following almost automorphic system:

$$
x^{\prime}(t)=A(t) x(t)+f(t)
$$

where $A(t)$ is an almost automorphic matrix function and $f(t)$ is an almost automorphic vector function.

Lemma 7 (see [27]). If the linear system (14) admits an exponential dichotomy, then system (16) has a unique almost automorphic solution:

$$
\begin{aligned}
x(t)= & \int_{-\infty}^{t} X(t) P X^{-1}(s) f(s) d s \\
& -\int_{t}^{+\infty} X(t)(I-P) X^{-1}(s) f(s) d s,
\end{aligned}
$$

where $X(t)$ is the fundamental solution matrix of (14).

Lemma 8 (see [33]). Let $c_{i}(t)$ be an almost automorphic function on $\mathbb{R}$ and

$$
M\left[c_{i}\right]=\lim _{T \rightarrow \infty} \frac{1}{T} \int_{t}^{t+T} c_{i}(s) d s>0, \quad i=1,2, \ldots, n
$$

then the linear system

$$
x^{\prime}(t)=\operatorname{diag}\left(-c_{1}(t),-c_{2}(t), \ldots,-c_{n}(t)\right) x(t)
$$

admits an exponential dichotomy on $\mathbb{R}$.

Definition 9. The almost automorphic solution $x^{*}(t)=$ $\left(x_{1}^{*}(t), x_{2}^{*}(t), \ldots, x_{n}^{*}(t)\right)^{T}$ of system (1) is said to be globally exponentially stable, if, for any solution $x(t)=$ $\left(x_{1}(t), x_{2}(t), \ldots, x_{n}(t)\right)^{T}$, there exist constants $M>0$ and $\mu>0$ such that, for all $t \in \mathbb{R}$,

$$
\left|x(t)-x^{*}(t)\right| \leq M e^{-\mu t}
$$

\section{Main Results}

In this section, we establish some results for the existence and uniqueness of almost automorphic solutions of (23).

Let $\mathbb{R}^{n}$ be the $n$-dimensional Euclidean space. Set $x=$ $\left\{x_{i}\right\}=\left(x_{1}, x_{2}, \ldots, x_{n}\right)^{T}$. For every $x \in \mathbb{R}^{n}$, its norm is defined by $\|x\|=\max _{1 \leq i \leq n}\left\{\omega_{i}^{-1}\left|x_{i}\right|\right\}$, where $\omega_{i}>0, i=1,2, \ldots, n$.

From $\left(H_{1}\right)$, the antiderivative of $1 / a_{i}\left(x_{i}\right)$ exists. We may choose an antiderivative $R_{i}\left(x_{i}\right)$ of $1 / a_{i}\left(x_{i}\right)$ with $R_{i}(0)=0$. Obviously, $R_{i}^{\prime}\left(x_{i}\right)=1 / a_{i}\left(x_{i}\right)$. By $a_{i}\left(x_{i}\right)>0$, we obtain that $R_{i}\left(x_{i}\right)$ is increasing about $x_{i}$ and the inverse function $R_{i}^{-1}\left(x_{i}\right)$ of $R_{i}\left(x_{i}\right)$ is existential, continuous, and differentiable. So, $\left(R_{i}^{-1}\right)^{\prime}\left(x_{i}\right)=a_{i}\left(x_{i}\right)$, where $\left(R_{i}^{-1}\right)^{\prime}\left(x_{i}\right)$ is the derivative of $R_{i}^{-1}\left(x_{i}\right)$ about $x_{i}$, and composition function $b_{i}\left(R_{i}^{-1}\left(x_{i}\right)\right)$ is differentiable. Denote $y_{i}(t)=R_{i}\left(x_{i}(t)\right)$. It is easy to see that
$y_{i}^{\prime}(t)=R_{i}^{\prime}\left(x_{i}(t)\right) x_{i}^{\prime}(t)=x_{i}^{\prime}(t) / a_{i}\left(x_{i}(t)\right)$ and $x_{i}(t)=R_{i}^{-1}\left(y_{i}(t)\right)$. Substituting these equalities into system (1), we can get that

$$
\begin{aligned}
y_{i}^{\prime}(t)= & -b_{i}\left(R_{i}^{-1}\left(y_{i}(t)\right)\right)+\sum_{j=1}^{n} c_{i j}(t) f_{j}\left(R_{j}^{-1}\left(y_{j}(t)\right)\right) \\
& +\sum_{j=1}^{n} d_{i j}(t) g_{j}\left(R_{j}^{-1}\left(y_{j}\left(t-\tau_{i j}(t)\right)\right)\right) \\
& +\sum_{j=1}^{n} e_{i j}(t) \mu_{j}(t)+\bigwedge_{j=1}^{n} \alpha_{i j}(t) \\
& \cdot \int_{-\infty}^{t} K_{i j}(t-s) h_{j}\left(R_{j}^{-1}\left(y_{j}(s)\right)\right) d s \\
& +\bigwedge_{j=1}^{n} T_{i j}(t) \mu_{j}(t)+\bigvee_{j=1}^{n} \beta_{i j}(t) \\
& \cdot \int_{-\infty}^{t} K_{i j}(t-s) h_{j}\left(R_{j}^{-1}\left(y_{j}(s)\right)\right) d s \\
& +\bigvee_{j=1}^{n} S_{i j}(t) \mu_{j}(t)+I_{i}(t), \\
& t>0, \quad i=1,2, \ldots, n .
\end{aligned}
$$

The initial condition of (21) is as follows:

$$
y_{i}(s)=R_{i}\left(\varphi_{i}(s)\right)=\phi_{i}(s), \quad s \in(-\infty, 0], i=1,2, \ldots, n .
$$

Then system (21) can be written as

$$
\begin{aligned}
y_{i}^{\prime}(t)= & -\widetilde{b}_{i}\left(y_{i}(t)\right) y_{i}(t)+\sum_{j=1}^{n} c_{i j}(t) f_{j}\left(R_{j}^{-1}\left(y_{j}(t)\right)\right) \\
& +\sum_{j=1}^{n} d_{i j}(t) g_{j}\left(R_{j}^{-1}\left(y_{j}\left(t-\tau_{i j}(t)\right)\right)\right) \\
& +\sum_{j=1}^{n} e_{i j}(t) \mu_{j}(t)+\bigwedge_{j=1}^{n} \alpha_{i j}(t) \\
& \cdot \int_{-\infty}^{t} K_{i j}(t-s) h_{j}\left(R_{j}^{-1}\left(y_{j}(s)\right)\right) d s \\
& +\bigwedge_{j=1}^{n} T_{i j}(t) \mu_{j}(t) \\
& +\bigvee_{j=1}^{n} \beta_{i j}(t) \int_{-\infty}^{t} K_{i j}(t-s) h_{j}\left(R_{j}^{-1}\left(y_{j}(s)\right)\right) d s \\
& +\bigvee_{j=1}^{n} S_{i j}(t) \mu_{j}(t)+I_{i}(t), \quad t>0,
\end{aligned}
$$

where $\widetilde{b}_{i}\left(y_{i}(t)\right) \triangleq b_{i}\left(R_{i}^{-1}\left(y_{i}(t)\right)\right) / y_{i}(t), i=1,2, \ldots, n$.

System (1) has an almost automorphic solution which is globally exponentially stable if and only if system (23) has an 
almost automorphic solution which is globally exponentially stable.

It is easy to see that

$$
\begin{aligned}
&\left|R_{i}^{-1}(u)-R_{i}^{-1}(v)\right|=\left|\left(R_{i}^{-1}\right)^{\prime}(\xi)(u-v)\right| \\
&=\left|a_{i}(\xi)\right||u-v| \leq a_{i}^{+}|u-v|, \\
& i=1,2, \ldots, n,
\end{aligned}
$$

where $\xi$ is between $u$ and $v$.

Lemma 10. Suppose that assumptions $\left(H_{3}\right),\left(H_{4}\right)$, and $\left(H_{5}\right)$ hold and $\varphi_{j} \in A A(\mathbb{R}, \mathbb{R})$; then

$$
\phi: t \longmapsto \int_{-\infty}^{t} K_{i j}(t-s) h_{j}\left(R_{j}^{-1}\left(\varphi_{j}(s)\right)\right) d s
$$

belongs to $A A(\mathbb{R}, \mathbb{R})$.

Proof. By Lemma 3, we have that, for $\varphi_{j} \in A A(\mathbb{R}, \mathbb{R})$, the function $\psi: s \mapsto h_{j}\left(R_{j}^{-1}\left(\varphi_{j}(s)\right)\right.$ belongs to $A A(\mathbb{R}, \mathbb{R})$. Now, let $\left\{s_{n}^{\prime}\right\}$ be a sequence of real numbers. By $\left(H_{5}\right)$ we can extract a subsequence $\left\{s_{n}\right\}$ of $\left\{s_{n}^{\prime}\right\}$ such that, for all $t, s \in \mathbb{R}$,

$$
\begin{gathered}
\lim _{n \rightarrow+\infty} K_{i j}\left(t-s+s_{n}\right)=K_{i j}^{1}(t-s), \\
\lim _{n \rightarrow+\infty} K_{i j}^{1}\left(t-s-s_{n}\right)=K_{i j}(t-s), \\
\lim _{n \rightarrow+\infty} \psi\left(t+s_{n}\right)=\psi^{1}(t), \quad \lim _{n \rightarrow+\infty} \psi^{1}\left(t-s_{n}\right)=\psi(t) .
\end{gathered}
$$

Set

$$
\phi^{1}: t \longmapsto \int_{-\infty}^{t} K_{i j}(t-s) \psi^{1}(s) d s
$$

Clearly,

$$
\begin{aligned}
\phi( & \left.+s_{n}\right)-\phi^{1}(t) \\
& =\int_{-\infty}^{t+s_{n}} K_{i j}\left(t-s-s_{n}\right) \psi(s) d s-\int_{-\infty}^{t} K_{i j}(t-s) \psi^{1}(s) d s \\
& =\int_{-\infty}^{t} K_{i j}(t-u) \psi\left(u+s_{n}\right) d u-\int_{-\infty}^{t} K_{i j}(t-s) \psi^{1}(s) d s \\
& =\int_{-\infty}^{t} K_{i j}(t-s)\left|\psi\left(s+s_{n}\right)-\psi^{1}(s)\right| d s \\
& \leq \int_{-\infty}^{t} \rho e^{-(t-s) C}\left|\psi\left(s+s_{n}\right)-\psi^{1}(s)\right| d s .
\end{aligned}
$$

By the well-known Lebesgue Dominated Convergence Theorem and $\left(H_{4}\right)$, we have, for all $t \in \mathbb{R}$,

$$
\lim _{n \rightarrow \infty} \phi\left(t+s_{n}\right)=\phi^{1}(t)
$$

Similarly, for each $t \in \mathbb{R}$,

$$
\lim _{n \rightarrow \infty} \phi^{1}\left(t-s_{n}\right)=\phi(t)
$$

which implies that $\phi: t \mapsto \int_{-\infty}^{t} K_{i j}(t-s) h_{j}\left(R_{j}^{-1}\left(\varphi_{j}(s)\right)\right) d s$ belongs to $A A(\mathbb{R}, \mathbb{R})$. The proof is complete.

Lemma 11. Suppose that assumptions $\left(H_{1}\right)-\left(H_{4}\right)$ hold. Define the nonlinear operator $\Phi$ by

$$
\Phi \varphi=\left((\Phi \varphi)_{1},(\Phi \varphi)_{2}, \ldots,(\Phi \varphi)_{n}\right)
$$

for each $\varphi \in A A\left(\mathbb{R}, \mathbb{R}^{n}\right)$, where for $i=1,2, \ldots, n$,

$$
\begin{aligned}
&(\Phi \varphi)_{i}(t) \\
&=\int_{-\infty}^{t} \exp \left(-\int_{s}^{t} \widetilde{b}_{i}\left(y_{i}(\theta)\right) d \theta\right) \\
& \cdot\left[\sum_{j=1}^{n} c_{i j}(s) f_{j}\left(R_{j}^{-1}\left(\varphi_{j}(s)\right)\right)\right. \\
&+\sum_{j=1}^{n} d_{i j}(s) g_{j}\left(R_{j}^{-1}\left(\varphi_{j}\left(s-\tau_{i j}(s)\right)\right)\right) \\
&+\sum_{j=1}^{n} e_{i j}(s) \mu_{j}(s) \\
&+\bigwedge_{j=1}^{n} \alpha_{i j}(s)
\end{aligned}
$$$$
\cdot \int_{-\infty}^{s} K_{i j}(s-u) h_{j}\left(R_{j}^{-1}\left(\varphi_{j}(u)\right)\right) d u
$$$$
+\bigwedge_{j=1}^{n} T_{i j}(t) \mu_{j}(t)
$$$$
+\bigvee_{j=1}^{n} \beta_{i j}(s)
$$$$
\cdot \int_{-\infty}^{s} K_{i j}(s-u) h_{j}\left(R_{j}^{-1}\left(\varphi_{j}(u)\right)\right) d u
$$

$$
\left.+\bigvee_{j=1}^{n} S_{i j}(s) \mu_{j}(s)+I_{i}(s)\right] d s .
$$

Then $\Phi$ maps $A A\left(\mathbb{R}, \mathbb{R}^{n}\right)$ into itself. 
Proof. First of all, let us check that $\Phi$ is well defined. Indeed, by Lemma 3 , the space $A A\left(\mathbb{R}, \mathbb{R}^{n}\right)$ is translation invariant. Besides, by Lemmas 5 and 10 , the function

$$
\begin{aligned}
\chi_{i} & : s \longmapsto \sum_{j=1}^{n} c_{i j}(s) f_{j}\left(R_{j}^{-1}\left(\varphi_{j}(s)\right)\right) \\
& +\sum_{j=1}^{n} d_{i j}(s) g_{j}\left(R_{j}^{-1}\left(\varphi_{j}\left(s-\tau_{i j}(s)\right)\right)\right)+\sum_{j=1}^{n} e_{i j}(s) \mu_{j}(s) \\
& +\bigwedge_{j=1}^{n} \alpha_{i j}(s) \int_{-\infty}^{s} K_{i j}(s-u) h_{j}\left(R_{j}^{-1}\left(\varphi_{j}(u)\right)\right) d u \\
& +\bigwedge_{j=1}^{n} T_{i j}(t) \mu_{j}(t) \\
& +\bigvee_{j=1}^{n} \beta_{i j}(s) \int_{-\infty}^{s} K_{i j}(s-u) h_{j}\left(R_{j}^{-1}\left(\varphi_{j}(u)\right)\right) d u \\
& +\bigvee_{j=1}^{n} S_{i j}(s) \mu_{j}(s)+I_{i}(s)
\end{aligned}
$$

belongs to $A A\left(\mathbb{R}, \mathbb{R}^{n}\right)$. Consequently we can write

$$
(\Phi \varphi)_{i}(t)=\int_{-\infty}^{t} \exp \left(-\int_{s}^{t} \widetilde{b}_{i}\left(y_{i}(\theta)\right) d \theta\right) \chi_{i}(s) d s .
$$

Let $\left\{s_{n}^{\prime}\right\}$ be a sequence of real numbers. By the composition theorem of almost automorphic functions, the function $\widetilde{b}_{i}\left(y_{i}(t)\right) \in A A\left(\mathbb{R}, \mathbb{R}^{n}\right)$. By $\left(H_{3}\right)$ we can extract a subsequence $\left\{s_{n}\right\}$ of $\left\{s_{n}^{\prime}\right\}$ such that, for all $t, s \in \mathbb{R}$,

$$
\begin{aligned}
\lim _{n \rightarrow+\infty} \widetilde{b}_{i}\left(y_{i}\left(t+s_{n}\right)\right) & =\widetilde{b}_{i}^{1}\left(y_{i}(t)\right), \\
\lim _{n \rightarrow+\infty} \widetilde{b}_{i}^{1}\left(y_{i}\left(t-s_{n}\right)\right) & =\widetilde{b}_{i}\left(y_{i}(t)\right), \\
\lim _{n \rightarrow+\infty} \chi_{i}\left(t+s_{n}\right) & =\chi_{i}^{1}(t), \\
\lim _{n \rightarrow+\infty} \chi_{i}^{1}\left(t-s_{n}\right) & =\chi_{i}(t) .
\end{aligned}
$$

Set

$$
\left(\Phi^{1} \varphi\right)_{i}(t)=\int_{-\infty}^{t} \exp \left(-\int_{s}^{t} \widetilde{b}_{i}^{1}\left(y_{i}(\theta)\right) d \theta\right) \chi_{i}^{1}(s) d s .
$$

Then it follows that

$$
\begin{gathered}
(\Phi \varphi)_{i}\left(t+s_{n}\right)-\left(\Phi^{1} \varphi\right)_{i}(t) \\
=\int_{-\infty}^{t+s_{n}} \exp \left(-\int_{s}^{t+s_{n}} \widetilde{b}_{i}\left(y_{i}(\theta)\right) d \theta\right) \chi_{i}(s) d s \\
\quad-\int_{-\infty}^{t} \exp \left(-\int_{s}^{t} \widetilde{b}_{i}^{1}\left(y_{i}(\theta)\right) d \theta\right) \chi_{i}^{1}(s) d s \\
=\int_{-\infty}^{t+s_{n}} \exp \left(-\int_{s-s_{n}}^{t} \widetilde{b}_{i}\left(y_{i}\left(\sigma+s_{n}\right)\right) d \sigma\right) \chi_{i}(s) d s \\
\quad-\int_{-\infty}^{t} \exp \left(-\int_{s}^{t} \widetilde{b}_{i}^{1}\left(y_{i}(\theta)\right) d \theta\right) \chi_{i}^{1}(s) d s
\end{gathered}
$$

$$
\begin{aligned}
= & \int_{-\infty}^{t} \exp \left(-\int_{u}^{t} \widetilde{b}_{i}\left(y_{i}\left(\sigma+s_{n}\right)\right) d \sigma\right) \chi_{i}\left(u+s_{n}\right) d u \\
& -\int_{-\infty}^{t} \exp \left(-\int_{s}^{t} \widetilde{b}_{i}^{1}\left(y_{i}(\theta)\right) d \theta\right) \chi_{i}^{1}(s) d s \\
= & \int_{-\infty}^{t} \exp \left(-\int_{u}^{t} \widetilde{b}_{i}\left(y_{i}\left(\sigma+s_{n}\right)\right) d \sigma\right) \chi_{i}\left(u+s_{n}\right) d u \\
& -\int_{-\infty}^{t} \exp \left(-\int_{u}^{t} \widetilde{b}_{i}\left(y_{i}\left(\sigma+s_{n}\right)\right) d \sigma\right) \chi_{i}^{1}(u) d u \\
& +\int_{-\infty}^{t} \exp \left(-\int_{u}^{t} \widetilde{b}_{i}\left(y_{i}\left(\sigma+s_{n}\right)\right) d \sigma\right) \chi_{i}^{1}(u) d u \\
& -\int_{-\infty}^{t} \exp \left(-\int_{u}^{t} \widetilde{b}_{i}^{1}\left(y_{i}(\theta)\right) d \theta\right) \chi_{i}^{1}(u) d u \\
= & \int_{-\infty}^{t} \exp \left(-\int_{s}^{t} \widetilde{b}_{i}\left(y_{i}\left(\sigma+s_{n}\right)\right) d \sigma\right) \\
& \cdot\left(\chi_{i}\left(s+s_{n}\right)-\chi_{i}^{1}(s)\right) d s \\
& +\int_{-\infty}^{t}\left(\exp \left(-\int_{s}^{t} \widetilde{b}_{i}\left(y_{i}\left(\sigma+s_{n}\right)\right) d \sigma\right)\right. \\
& \left.\quad-\exp \left(-\int_{s}^{t} \widetilde{b}_{i}^{1}\left(y_{i}(\theta)\right) d \theta\right)\right) \chi_{i}^{1}(s) d s .
\end{aligned}
$$

By the Lebesgue Dominated Convergence Theorem we obtain immediately that

$$
\lim _{n \rightarrow+\infty}(\Phi \varphi)_{i}\left(t+s_{n}\right)=\left(\Phi^{1} \varphi\right)_{i}(t)
$$

for all $t \in \mathbb{R}$.

Reasoning in a similar way to the first step we can show easily that

$$
\lim _{n \rightarrow+\infty}\left(\Phi^{1} \varphi\right)_{i}\left(t-s_{n}\right)=(\Phi \varphi)_{i}(t)
$$

for all $t \in \mathbb{R}$. Consequently, the function $\Phi \varphi$ belongs to $A A\left(\mathbb{R}, \mathbb{R}^{n}\right)$.

Theorem 12. Assume that $\left(H_{1}\right)-\left(H_{5}\right)$ hold, then system (23) has a unique almost automorphic solution in the region

$$
\mathscr{B}=B\left(\varphi^{0}, r\right)=\left\{\varphi \in A A\left(\mathbb{R}, \mathbb{R}^{n}\right),\left\|\varphi-\varphi^{0}\right\| \leq \frac{r J}{1-r}\right\},
$$

if the following condition holds

$$
\begin{aligned}
r=\max _{1 \leq i \leq n}\{( & \left(\lambda_{i} a_{i}^{-} \omega_{i}^{-1}\right)^{-1} \\
& {\left[\sum_{j=1}^{n} c_{i j}^{+} L_{j}^{f} a_{j}^{+} \omega_{j}+\sum_{j=1}^{n} d_{i j}^{+} L_{j}^{g} a_{j}^{+} \omega_{j}\right.} \\
& \left.\left.+\sum_{j=1}^{n}\left(\alpha_{i j}^{+}+\beta_{i j}^{+}\right) L_{j}^{h} a_{j}^{+} \omega_{j}\right]\right\}<1,
\end{aligned}
$$


where $\varphi^{0}=\left(\varphi_{1}^{0}, \varphi_{1}^{0}, \ldots, \varphi_{1}^{0}\right)^{T}$,

$$
\begin{array}{r}
\varphi_{i}^{0}(t)=\int_{-\infty}^{t} J_{i}(s) \exp \left(-\int_{s}^{t} \widetilde{b}_{i}\left(y_{i}(\theta)\right) d \theta\right) d s \\
i=1,2, \ldots, n .
\end{array}
$$

Proof. Obviously, $\mathscr{B}$ is a closed convex subset of $A A\left(\mathbb{R}, \mathbb{R}^{n}\right)$ and one has

$$
\begin{aligned}
& \left\|\varphi^{0}\right\| \\
& \quad=\max _{1 \leq i \leq n} \sup _{t \in \mathbb{R}}\left\{\omega_{i}^{-1}\left|\int_{-\infty}^{t} J_{i}(s) \exp \left(-\int_{s}^{t} \widetilde{b}_{i}\left(y_{i}(\theta)\right) d \theta\right) d s\right|\right\} \\
& \quad \leq \max _{1 \leq i \leq n} \sup _{t \in \mathbb{R}}\left\{\omega_{i}^{-1} \int_{-\infty}^{t}\left|J_{i}(s)\right| \exp \left(-\int_{s}^{t} \widetilde{b}_{i}\left(y_{i}(\theta)\right) d \theta\right) d s\right\} \\
& \quad \leq \max _{1 \leq i \leq n} \sup _{t \in \mathbb{R}}\left\{\omega_{i}^{-1} \int_{-\infty}^{t} J_{i}^{+} \exp \left(\lambda_{i} a_{i}^{-}(s-t)\right) d s\right\} \\
& \quad=\max _{1 \leq i \leq n}\left\{\omega_{i}^{-1} \frac{J_{i}^{+}}{\lambda_{i} a_{i}^{-}}\right\}=J .
\end{aligned}
$$

Thus, for any $\varphi \in \mathscr{B}$, we have

$$
\|\varphi\| \leq\left\|\varphi-\varphi^{0}\right\|+\left\|\varphi^{0}\right\| \leq \frac{r J}{1-r}+J=\frac{1}{1-r} .
$$

Now we prove that $\Phi$ is a self-mapping from $\mathscr{B}$ to $\mathscr{B}$. In fact, for arbitrary $\varphi \in \mathscr{B}$, we have

$$
\begin{aligned}
&\left\|\Phi \varphi-\varphi^{0}\right\| \max _{1 \leq i \leq n} \sup _{t \in \mathbb{R}}\left\{\omega_{i}^{-1} \mid \int_{-\infty}^{t} \exp \left(-\int_{s}^{t} \widetilde{b}_{i}\left(y_{i}(\theta)\right) d \theta\right)\right. \\
& \cdot {\left[\sum_{j=1}^{n} c_{i j}(s) f_{j}\left(R_{j}^{-1}\left(\varphi_{j}(s)\right)\right)\right.} \\
&+ \sum_{j=1}^{n} d_{i j}(s) g_{j} \\
& \cdot\left(R_{j}^{-1}\left(\varphi_{j}\left(s-\tau_{i j}(s)\right)\right)\right) \\
&+ \bigwedge_{j=1}^{n} \alpha_{i j}(s) \\
& \cdot \int_{-\infty}^{s} K_{i j}(s-u) h_{j} \\
& \cdot\left(R_{j}^{-1}\left(\varphi_{j}(u)\right)\right) d u \\
&+\bigvee_{j=1}^{n} \beta_{i j}(s)
\end{aligned}
$$

$$
\begin{aligned}
& \cdot \int_{-\infty}^{s} K_{i j}(s-u) h_{j} \\
& \left.\left.\cdot\left(R_{j}^{-1}\left(\varphi_{j}(u)\right)\right) d u\right] d s \mid\right\}
\end{aligned}
$$$$
\leq \max _{1 \leq i \leq n} \sup _{t \in \mathbb{R}}\left\{\omega_{i}^{-1} \int_{-\infty}^{t} \exp \left(-\int_{s}^{t} \widetilde{b}_{i}\left(y_{i}(\theta)\right) d \theta\right)\right.
$$$$
\cdot\left[\sum_{j=1}^{n}\left|c_{i j}(s)\right|\left|f_{j}\left(R_{j}^{-1}\left(\varphi_{j}(s)\right)\right)\right|\right.
$$$$
+\sum_{j=1}^{n}\left|d_{i j}(s)\right|
$$$$
\cdot\left|g_{j}\left(R_{j}^{-1}\left(\varphi_{j}\left(s-\tau_{i j}(s)\right)\right)\right)\right|
$$$$
+\bigwedge_{j=1}^{n}\left|\alpha_{i j}(s)\right|
$$$$
\cdot \int_{-\infty}^{s} K_{i j}(s-u)
$$$$
\cdot\left|h_{j}\left(R_{j}^{-1}\left(\varphi_{j}(u)\right)\right)\right| d u
$$$$
+\bigvee_{j=1}^{n}\left|\beta_{i j}(s)\right|
$$$$
\cdot \int_{-\infty}^{s} K_{i j}(s-u)
$$$$
\left.\left.\cdot\left|h_{j}\left(R_{j}^{-1}\left(\varphi_{j}(u)\right)\right)\right| d u\right] d s\right\}
$$$$
\leq \max _{1 \leq i \leq n} \sup _{t \in \mathbb{R}}\left\{\omega_{i}^{-1} \int_{-\infty}^{t} \exp \left(-\int_{s}^{t} \widetilde{b}_{i}\left(y_{i}(\theta)\right) d \theta\right)\right.
$$$$
\cdot\left[\sum_{j=1}^{n} c_{i j}^{+} L_{j}^{f} a_{j}^{+}\left|\varphi_{j}(s)\right|\right.
$$$$
+\sum_{j=1}^{n} d_{i j}^{+} L_{j}^{g} a_{j}^{+}\left|\varphi_{j}\left(s-\tau_{i j}(s)\right)\right|
$$$$
+\sum_{j=1}^{n}\left(\alpha_{i j}^{+}+\beta_{i j}^{+}\right) L_{j}^{h} a_{j}^{+}
$$$$
\left.\left.\cdot \int_{-\infty}^{s} K_{i j}(s-u)\left|\varphi_{j}(u)\right| d u\right] d s\right\}
$$$$
\leq \max _{1 \leq i \leq n} \sup _{t \in \mathbb{R}}\left\{\omega_{i}^{-1} \int_{-\infty}^{t} \exp \left(-\int_{s}^{t} \widetilde{b}_{i}\left(y_{i}(\theta)\right) d \theta\right)\right.
$$ 


$$
\begin{array}{r}
\cdot\left[\sum_{j=1}^{n} c_{i j}^{+} L_{j}^{f} a_{j}^{+} \omega_{j}+\sum_{j=1}^{n} d_{i j}^{+} L_{j}^{g} a_{j}^{+} \omega_{j}\right. \\
\left.\left.\quad+\sum_{j=1}^{n}\left(\alpha_{i j}^{+}+\beta_{i j}^{+}\right) L_{j}^{h} a_{j}^{+} \omega_{j}\right] d s\right\}
\end{array}
$$

$\cdot\|\varphi\|$

$$
\begin{aligned}
& \leq \max _{1 \leq i \leq n} \sup _{t \in \mathbb{R}}\left\{\omega_{i}^{-1} \int_{-\infty}^{t}\right. \exp \left(-\lambda_{i} a_{i}^{-}(t-s)\right) \\
& \cdot\left[\sum_{j=1}^{n} c_{i j}^{+} L_{j}^{f} a_{j}^{+} \omega_{j}+\sum_{j=1}^{n} d_{i j}^{+} L_{j}^{g} a_{j}^{+} \omega_{j}\right. \\
&\left.\left.+\sum_{j=1}^{n}\left(\alpha_{i j}^{+}+\beta_{i j}^{+}\right) L_{j}^{h} a_{j}^{+} \omega_{j}\right] d s\right\}
\end{aligned}
$$

$\cdot\|\varphi\|$

$$
\begin{aligned}
=\max _{1 \leq i \leq n}\{ & \left(\lambda_{i} a_{i}^{-} \omega_{i}^{-1}\right)^{-1} \\
& {\left[\sum_{j=1}^{n} c_{i j}^{+} L_{j}^{f} a_{j}^{+} \omega_{j}+\sum_{j=1}^{n} d_{i j}^{+} L_{j}^{g} a_{j}^{+} \omega_{j}\right.} \\
& \left.\left.+\sum_{j=1}^{n}\left(\alpha_{i j}^{+}+\beta_{i j}^{+}\right) L_{j}^{h} a_{j}^{+} \omega_{j}\right]\right\}
\end{aligned}
$$

$\cdot\|\varphi\|$

$=r\|\varphi\| \leq \frac{r M}{1-r}$,

which implies that $\Phi \varphi \in \mathscr{B}$, so the mapping $\Phi$ is a selfmapping from $\mathscr{B}$ to $\mathscr{B}$. Next, we will prove that $\Phi$ is a contraction mapping. For any $\varphi, \psi \in \mathscr{B}$, we have that

$$
\begin{gathered}
\|\Phi \varphi-\Phi \psi\| \\
=\max _{1 \leq i \leq n} \sup _{t \in \mathbb{R}}\left\{\omega_{i}^{-1} \mid \int_{-\infty}^{t} \exp \left(-\int_{s}^{t} \widetilde{b}_{i}\left(y_{i}(\theta)\right) d \theta\right)\right. \\
\cdot\left[\sum_{j=1}^{n} c_{i j}(s)\right. \\
\cdot\left[f_{j}\left(R_{j}^{-1}\left(\varphi_{j}(s)\right)\right)\right. \\
\left.-f_{j}\left(R_{j}^{-1}\left(\psi_{j}(s)\right)\right)\right] \\
+\sum_{j=1}^{n} d_{i j}(s)
\end{gathered}
$$

$$
\begin{gathered}
\cdot\left[g_{j}\left(R_{j}^{-1}\left(\varphi_{j}\left(s-\tau_{i j}(s)\right)\right)\right)\right. \\
\left.-g_{j}\left(R_{j}^{-1}\left(\psi_{j}\left(s-\tau_{i j}(s)\right)\right)\right)\right] \\
+\bigwedge_{j=1}^{n} \alpha_{i j}(s) \\
\cdot\left[\int_{-\infty}^{s} K_{i j}(s-u) h_{j}\right. \\
\cdot\left(R_{j}^{-1}\left(\varphi_{j}(u)\right)\right) d u \\
-\int_{-\infty}^{s} K_{i j}(s-u) h_{j} \\
+\bigvee_{j=1}^{n} \beta_{i j}(s) \\
\cdot\left[\int_{-\infty}^{s} K_{i j}(s-u) h_{j}\right.
\end{gathered}
$$$$
\cdot\left(R_{j}^{-1}\left(\varphi_{j}(u)\right)\right) d u
$$$$
-\int_{-\infty}^{s} K_{i j}(s-u) h_{j}
$$$$
\left.\left.\left.\cdot\left(R_{j}^{-1}\left(\psi_{j}(u)\right)\right) d u\right]\right] d s \mid\right\}
$$$$
\leq \max _{1 \leq i \leq n} \sup _{t \in \mathbb{R}}\left\{\omega_{i}^{-1} \int_{-\infty}^{t} \exp \left(-\int_{s}^{t} \widetilde{b}_{i}\left(y_{i}(\theta)\right) d \theta\right)\right.
$$$$
\cdot\left[\sum_{j=1}^{n} c_{i j}^{+} L_{j}^{f} a_{j}^{+}\left|\varphi_{j}(s)-\psi_{j}(s)\right|\right.
$$$$
+\sum_{j=1}^{n} d_{i j}^{+} L_{j}^{g} a_{j}^{+}
$$$$
\cdot\left|\varphi_{j}\left(s-\tau_{i j}(s)\right)-\psi_{j}\left(s-\tau_{i j}(s)\right)\right|
$$$$
+\sum_{j=1}^{n}\left(\alpha_{i j}^{+}+\beta_{i j}^{+}\right) L_{j}^{h} a_{j}^{+}
$$$$
\cdot \int_{-\infty}^{s} K_{i j}(s-u)
$$$$
\left.\left.\cdot\left|\varphi_{j}(u)-\psi_{j}(u)\right| d u\right] d s\right\}
$$$$
\leq \max _{1 \leq i \leq n} \sup _{t \in \mathbb{R}}\left\{\omega_{i}^{-1} \int_{-\infty}^{t} \exp \left(-\int_{s}^{t} \widetilde{b}_{i}\left(y_{i}(\theta)\right) d \theta\right)\right.
$$$$
\cdot\left[\sum_{j=1}^{n} c_{i j}^{+} L_{j}^{f} a_{j}^{+} \omega_{j}+\sum_{j=1}^{n} d_{i j}^{+} L_{j}^{g} a_{j}^{+} \omega_{j}\right.
$$ 


$$
\begin{array}{r}
\left.\left.+\sum_{j=1}^{n}\left(\alpha_{i j}^{+}+\beta_{i j}^{+}\right) L_{j}^{h} a_{j}^{+} \omega_{j}\right] d s\right\} \\
\qquad \max _{1 \leq i \leq n} \sup _{t \in \mathbb{R}}\left\{\omega_{i}^{-1} \int_{-\infty}^{t} \exp \left(-\lambda_{i} a_{i}^{-}(t-s)\right)\right. \\
\cdot\left[\sum_{j=1}^{n} c_{i j}^{+} L_{j}^{f} a_{j}^{+} \omega_{j}+\sum_{j=1}^{n} d_{i j}^{+} L_{j}^{g} a_{j}^{+} \omega_{j}\right. \\
\left.\left.\cdot\|\varphi-\psi\| \sum_{j=1}^{n}\left(\alpha_{i j}^{+}+\beta_{i j}^{+}\right) L_{j}^{h} a_{j}^{+} \omega_{j}\right] d s\right\} \\
=\max _{1 \leq i \leq n}\left\{\left(\lambda_{i} a_{i}^{-} \omega_{i}^{-1}\right)^{-1}\right. \\
\cdot\left[\sum_{j=1}^{n} c_{i j}^{+} L_{j}^{f} a_{j}^{+} \omega_{j}+\sum_{j=1}^{n} d_{i j}^{+} L_{j}^{g} a_{j}^{+} \omega_{j}\right. \\
\left.\left.+\sum_{j=1}^{n}\left(\alpha_{i j}^{+}+\beta_{i j}^{+}\right) L_{j}^{h} a_{j}^{+} \omega_{j}\right]\right\}\|\varphi-\psi\| \\
=r\|\varphi-\psi\| .
\end{array}
$$

Because $r<1$, so $\Phi$ is a contraction mapping. Therefore, $\Phi$ has a unique fixed point $x^{*} \in \mathscr{B}$ such that $\Phi\left(x^{*}\right)=x^{*}$; that is, (23) has a unique almost automorphic solution $x^{*} \in \mathscr{B}$. This completes the proof of Theorem 12.

Theorem 13. Assume that $\left(H_{1}\right)-\left(H_{5}\right)$ hold, the almost automorphic solution of (23) is globally exponentially stable if, for $i=1,2, \ldots, n$, the following conditions hold

$$
\begin{aligned}
\lambda_{i} a_{i}^{-} \omega_{i}>\sum_{j=1}^{n}( & c_{i j}^{+} L_{j}^{f} a_{j}^{+} \omega_{j}+d_{i j}^{+} L_{j}^{g} a_{j}^{+} \omega_{j} \\
& \left.+\left(\alpha_{i j}^{+}+\beta_{i j}^{+}\right) L_{j}^{h} a_{j}^{+} \omega_{j} \int_{0}^{+\infty} K_{i j}(s) d s\right) .
\end{aligned}
$$

Proof. For $i=1,2, \ldots, n$, consider the function $\Theta_{i}(\gamma)$ given by

$$
\begin{aligned}
\Theta_{i}(\gamma)= & \gamma-\lambda_{i} a_{i}^{-}+\omega_{i}^{-1} \sum_{j=1}^{n} c_{i j}^{+} L_{j}^{f} a_{j}^{+} \omega_{j} \\
& +\omega_{i}^{-1} \sum_{j=1}^{n} d_{i j}^{+} L_{j}^{g} a_{j}^{+} \omega_{j} e^{\gamma \tau} \\
& +\omega_{i}^{-1} \sum_{j=1}^{n}\left(\alpha_{i j}^{+}+\beta_{i j}^{+}\right) L_{j}^{h} a_{j}^{+} \omega_{j} \int_{0}^{+\infty} K_{i j}(s) e^{\gamma s} d s .
\end{aligned}
$$

It is clear that the function $\Theta_{i}(\gamma)$ is continuous on $\mathbb{R}^{+}$and by (47), we have

$$
\begin{aligned}
\Theta_{i}(0)= & -\lambda_{i} a_{i}^{-}+\omega_{i}^{-1} \sum_{j=1}^{n} c_{i j}^{+} L_{j}^{f} a_{j}^{+} \omega_{j} \\
& +\omega_{i}^{-1} \sum_{j=1}^{n} d_{i j}^{+} L_{j}^{g} a_{j}^{+} \omega_{j} \\
& +\omega_{i}^{-1} \sum_{j=1}^{n}\left(\alpha_{i j}^{+}+\beta_{i j}^{+}\right) L_{j}^{h} a_{j}^{+} \omega_{j} \int_{0}^{+\infty} K_{i j}(s) d s \\
< & 0, \quad i=1,2, \ldots, n .
\end{aligned}
$$

Thus, there exists a sufficiently small constant $\mu$ such that

$$
\Theta_{i}(\mu)<0, \quad i=1,2, \ldots, n .
$$

From Theorem 12, we see that system (23) has at least one almost automorphic solution $y^{*}(t)=\left\{y_{i}^{*}(t)\right\}_{i=1}^{n}$ with initial condition $y_{i}^{*}(s)=\phi_{i}^{*}(s), s \in(-\infty, 0], i=1,2, \ldots, n$. Suppose that $y(t)=\left\{y_{i}(t)\right\}_{i=1}^{n}$ is an arbitrary solution with initial condition $y_{i}(s)=\phi_{i}(s), s \in(-\infty, 0], i=1,2, \ldots, n$. Set $z(t)=y(t)-y^{*}(t)$; then it follows from system (23) that

$$
\begin{aligned}
& z_{i}^{\prime}(t) \\
& =-\left[b_{i}\left(R_{i}^{-1}\left(z_{i}(t)+y_{i}^{*}(t)\right)\right)-b_{i}\left(R_{i}^{-1}\left(y_{i}^{*}(t)\right)\right)\right] \\
& +\sum_{j=1}^{n} c_{i j}(t)\left[f_{j}\left(R_{j}^{-1}\left(z_{j}(t)+y_{j}^{*}(t)\right)\right)\right. \\
& \left.-f_{j}\left(R_{j}^{-1}\left(y_{j}^{*}(t)\right)\right)\right] \\
& +\sum_{j=1}^{n} d_{i j}(t)\left[g_{j}\left(R_{j}^{-1}\left(z_{j}\left(t-\tau_{i j}(t)\right)+y_{j}^{*}\left(t-\tau_{i j}(t)\right)\right)\right)\right. \\
& \left.-g_{j}\left(R_{j}^{-1}\left(y_{j}^{*}\left(t-\tau_{i j}(t)\right)\right)\right)\right] \\
& +\bigwedge_{j=1}^{n} \alpha_{i j}(t) \\
& \cdot \int_{-\infty}^{t} K_{i j}(t-s)\left[h_{j}\left(R_{j}^{-1}\left(z_{j}(s)+y_{j^{*}}(s)\right)\right)\right. \\
& \left.-h_{j}\left(R_{j}^{-1}\left(y_{j}^{*}(s)\right)\right)\right] d s \\
& +\bigvee_{j=1}^{n} \beta_{i j}(t) \\
& \cdot \int_{-\infty}^{t} K_{i j}(t-s)\left[h_{j}\left(R_{j}^{-1}\left(z_{j}(s)+y_{j^{*}}(s)\right)\right)\right. \\
& \left.-h_{j}\left(R_{j}^{-1}\left(y_{j}^{*}(s)\right)\right)\right] d s, \\
& t>0 \text {, }
\end{aligned}
$$


where $i=1,2, \ldots, n$. The initial condition of (51) is

$$
\begin{gathered}
z_{i}(s)=\psi_{i}(s)=\phi_{i}(s)-\phi_{i}^{*}(s), \\
s \in(-\infty, 0], \quad i=1,2, \ldots, n .
\end{gathered}
$$

Similar to system (23), we set $\widehat{b}_{i}\left(z_{i}(t)\right) \triangleq\left(b_{i}\left(R_{i}^{-1}\left(z_{i}(t)+\right.\right.\right.$ $\left.\left.\left.y_{i}^{*}(t)\right)\right)-b_{i}\left(R_{i}^{-1}\left(y_{i}^{*}(t)\right)\right)\right) / z_{i}(t)$. In view of $\left(H_{2}\right)$ and $(24)$, we have $\widehat{b}_{i}\left(R_{i}^{-1}(\cdot)\right) \geq \lambda_{i} a_{i}^{-}>0$.

Now, we consider functions $u_{i}(t)=\omega_{i}^{-1} e^{\mu t}\left|y_{i}(t)-y_{i}^{*}(t)\right|=$ $\omega_{i}^{-1} e^{\mu t}\left|z_{i}(t)\right|, \mu>0, i=1,2, \ldots, n$. Then

$$
\begin{aligned}
& 0 \leq D^{+} u_{i}(t)=\omega_{i}^{-1} e^{\mu t}\left\{\mu\left|z_{i}(t)\right|+\frac{d\left|z_{i}(t)\right|}{d t}\right\} \\
& =\omega_{i}^{-1} e^{\mu t} \\
& \text {. }\left\{\mu\left|z_{i}(t)\right|+\operatorname{sgn}\left(z_{i}(t)\right)\right. \\
& \cdot\left[-\widehat{b}_{i}\left(z_{i}(t)\right) z_{i}(t)+\sum_{j=1}^{n} c_{i j}(t)\right. \\
& \cdot\left[f_{j}\left(R_{j}^{-1}\left(z_{j}(t)+y_{j}^{*}(t)\right)\right)-f_{j}\left(R_{j}^{-1}\left(y_{j}^{*}(t)\right)\right)\right] \\
& +\sum_{j=1}^{n} d_{i j}(t) \\
& \cdot\left[g_{j}\left(R_{j}^{-1}\left(z_{j}\left(t-\tau_{i j}(t)\right)+y_{j}^{*}\left(t-\tau_{i j}(t)\right)\right)\right)\right. \\
& \left.-g_{j}\left(R_{j}^{-1}\left(y_{j}^{*}\left(t-\tau_{i j}(t)\right)\right)\right)\right]+\bigwedge_{j=1}^{n} \alpha_{i j}(t) \\
& \cdot \int_{-\infty}^{t} K_{i j}(t-s)\left[h_{j}\left(R_{j}^{-1}\left(z_{j}(s)+y_{j^{*}}(s)\right)\right)\right. \\
& \left.-h_{j}\left(R_{j}^{-1}\left(y_{j}^{*}(s)\right)\right)\right] d s
\end{aligned}
$$

$$
\begin{aligned}
&+ \bigvee_{j=1}^{n} \beta_{i j}(t) \\
& \cdot \int_{-\infty}^{t} K_{i j}(t-s)\left[h_{j}\left(R_{j}^{-1}\left(z_{j}(s)+y_{j^{*}}(s)\right)\right)\right. \\
&\left.\left.\left.-h_{j}\left(R_{j}^{-1}\left(y_{j}^{*}(s)\right)\right)\right] d s\right]\right\}
\end{aligned}
$$

$\leq \omega_{i}^{-1} e^{\mu t}$

$\cdot\left\{\mu\left|z_{i}(t)\right|-\lambda_{i} a_{i}^{-}\left|z_{i}(t)\right|\right.$

$+\sum_{j=1}^{n} c_{i j}^{+}\left|f_{j}\left(R_{j}^{-1}\left(z_{j}(t)+y_{j}^{*}(t)\right)\right)-f_{j}\left(R_{j}^{-1}\left(y_{j}^{*}(t)\right)\right)\right|$

$$
\begin{gathered}
+\sum_{j=1}^{n} d_{i j}^{+} \mid g_{j}\left(R_{j}^{-1}\left(z_{j}\left(t-\tau_{i j}(t)\right)+y_{j}^{*}\left(t-\tau_{i j}(t)\right)\right)\right) \\
-g_{j}\left(R_{j}^{-1}\left(y_{j}^{*}\left(t-\tau_{i j}(t)\right)\right)\right) \mid
\end{gathered}
$$$$
+\bigwedge_{j=1}^{n} \alpha_{i j}^{+}
$$$$
\cdot \int_{-\infty}^{t} K_{i j}(t-s) \mid h_{j}\left(R_{j}^{-1}\left(z_{j}(s)+y_{j^{*}}(s)\right)\right)
$$$$
-h_{j}\left(R_{j}^{-1}\left(y_{j}^{*}(s)\right)\right) \mid d s
$$

$+\bigvee_{j=1}^{n} \beta_{i j}^{+}$

$$
\begin{array}{r}
\cdot \int_{-\infty}^{t} K_{i j}(t-s) \mid h_{j}\left(R_{j}^{-1}\left(z_{j}(s)+y_{j^{*}}(s)\right)\right) \\
\left.-h_{j}\left(R_{j}^{-1}\left(y_{j}^{*}(s)\right)\right) \mid d s\right\}
\end{array}
$$

$$
\leq \omega_{i}^{-1} e^{\mu t}
$$$$
\left\{\mu\left|z_{i}(t)\right|-\lambda_{i} a_{i}^{-}\left|z_{i}(t)\right|\right.
$$$$
+\sum_{j=1}^{n} c_{i j}^{+} L_{j}^{f} a_{j}^{+}\left|z_{j}(t)\right|+\sum_{j=1}^{n} d_{i j}^{+} L_{j}^{g} a_{j}^{+}\left|z_{j}\left(t-\tau_{i j}(t)\right)\right|
$$

$$
\left.+\sum_{j=1}^{n}\left(\alpha_{i j}^{+}+\beta_{i j}^{+}\right) L_{j}^{h} a_{j}^{+} \int_{-\infty}^{t} K_{i j}(t-s)\left|z_{j}(s)\right| d s\right\}
$$$$
\leq-\left(\lambda_{i} a_{i}^{-}-\mu\right) u_{i}(t)+\omega_{i}^{-1} \sum_{j=1}^{n} c_{i j}^{+} L_{j}^{f} a_{j}^{+} \omega_{j} u_{j}(t)
$$$$
+\omega_{i}^{-1} \sum_{j=1}^{n} d_{i j}^{+} L_{j}^{g} a_{j}^{+} \omega_{j} e^{\mu \tau} u_{j}\left(t-\tau_{i j}(t)\right)
$$

$$
+\omega_{i}^{-1} \sum_{j=1}^{n}\left(\alpha_{i j}^{+}+\beta_{i j}^{+}\right) L_{j}^{h} a_{j}^{+} \omega_{j}
$$

$$
\cdot \int_{-\infty}^{t} K_{i j}(t-s) e^{\mu(t-s)} u_{j}(s) d s
$$

$$
\leq-\left(\lambda_{i} a_{i}^{-}-\mu\right) u_{i}(t)+\omega_{i}^{-1} \sum_{j=1}^{n} c_{i j}^{+} L_{j}^{f} a_{j}^{+} \omega_{j} u_{j}(t)
$$$$
+\omega_{i}^{-1} \sum_{j=1}^{n} d_{i j}^{+} L_{j}^{g} a_{j}^{+} \omega_{j} e^{\mu \tau} \sup _{t-\tau \leq s \leq t} u_{j}(s)
$$$$
+\omega_{i}^{-1} \sum_{j=1}^{n}\left(\alpha_{i j}^{+}+\beta_{i j}^{+}\right) L_{j}^{h} a_{j}^{+} \omega_{j}
$$

$$
\cdot \int_{0}^{+\infty} K_{i j}(s) e^{\mu s} d s \sup _{s \leq t} u_{j}(s)
$$

$$
i=1,2, \ldots, n \text {. }
$$


Set $M=\max _{1 \leq i \leq n} \sup _{t \in(-\infty, 0]}\left\{\omega_{i}^{-1}\left|z_{i}(t)\right|\right\}=$ $\max _{1 \leq i \leq n} \sup _{t \in(-\infty, 0]}\left\{\omega_{i}^{-1}\left|\phi_{i}(t)-\phi_{i}^{*}(t)\right|\right\}$ and $\epsilon>0$ is an arbitrary real number. Then, we can get

$$
\begin{aligned}
u_{i}(t) & =\omega_{i}^{-1} e^{\mu t}\left|z_{i}(t)\right| \leq \omega_{i}^{-1}\left|z_{i}(t)\right| \\
& \leq M \leq M+\epsilon, \\
& t \in(-\infty, 0], \quad i=1,2, \ldots, n .
\end{aligned}
$$

In the following, we will show that

$$
u_{i}(t)<M+\epsilon, \quad t \in(0,+\infty), i=1,2, \ldots, n .
$$

If (55) is not true, without loss of generality, then there exist a constant $l$ and a first time $t_{1}$ such that

$$
\begin{gathered}
u_{i}(t)<M+\epsilon, \quad i \neq l, t \in\left(-\infty, t_{1}\right], \\
u_{l}(t)<M+\epsilon, \quad t \in\left(-\infty, t_{1}\right), \\
u_{l}\left(t_{1}\right)=M+\epsilon .
\end{gathered}
$$

Therefore, we have

$$
\begin{aligned}
D^{+} u_{l}\left(t_{1}\right) & \\
\leq & -\left(\lambda_{l} a_{l}^{-}-\mu\right) u_{l}\left(t_{1}\right)+\omega_{l}^{-1} \sum_{j=1}^{n} c_{l j}^{+} L_{j}^{f} a_{j}^{+} \omega_{j} u_{j}\left(t_{1}\right) \\
& +\omega_{l}^{-1} \sum_{j=1}^{n} d_{l j}^{+} L_{j}^{g} a_{j}^{+} \omega_{j} e^{\mu \tau} \sup _{t_{1}-\tau \leq s \leq t_{1}} u_{j}(s) \\
& +\omega_{l}^{-1} \sum_{j=1}^{n}\left(\alpha_{l j}^{+}+\beta_{l j}^{+}\right) L_{j}^{h} a_{j}^{+} \omega_{j} \\
& \cdot \int_{0}^{+\infty} K_{l j}(s) e^{\mu s} d s \sup _{s \leq t} u_{j}(s) \\
< & \left\{\begin{array}{l}
\mu-\lambda_{l} a_{l}^{-}+\omega_{l}^{-1} \sum_{j=1}^{n} c_{l j}^{+} L_{j}^{f} a_{j}^{+} \omega_{j} \\
+
\end{array}\right. \\
& +\Theta_{l}(\mu)\left(M+\varepsilon+\omega_{l}^{-1} \sum_{j=1}^{n} d_{l j}^{+} L_{j}^{g} a_{j}^{+} \omega_{j} e^{\mu \tau} \sum_{j=1}^{n}\left(\alpha_{l j}^{+}+\beta_{l j}^{+}\right) L_{j}^{h} a_{j}^{+} \omega_{j} \int_{0}^{+\infty} K_{l j}(s) e^{\mu s} d s\right\} \\
& +0 .
\end{aligned}
$$

This is a contradiction; hence (55) holds. Let $\epsilon \rightarrow 0^{+}$, then we have

$$
u_{i}(t)<M, \quad t \in(0,+\infty), \quad i=1,2, \ldots, n .
$$

Together, (54) with (58) give the following:

$$
u_{i}(t)<M, \quad t \in \mathbb{R}, i=1,2, \ldots, n .
$$

Then, we have

$$
\left|z_{i}(t)\right|=\left|y_{i}(t)-y_{i}^{*}(t)\right| \leq M \omega_{i} e^{-\mu t}, \quad i=1,2, \ldots, n,
$$

which means that the almost automorphic solution of the system (23) is globally exponentially stable. The proof of Theorem 13 is completed.

\section{An Example}

In this section, we give an example to illustrate the feasibility and effectiveness of our results obtained in Section 3.

Example 1. Let $n=2$. Consider the following system:

$$
\begin{aligned}
x_{i}^{\prime}(t)=- & a_{i}\left(x_{i}(t)\right) \\
& \cdot\left[b_{i}\left(x_{i}(t)\right)-\sum_{j=1}^{2} c_{i j}(t) f_{j}\left(x_{j}(t)\right)\right. \\
& -\sum_{j=1}^{2} d_{i j}(t) g_{j}\left(x_{j}\left(t-\tau_{i j}(t)\right)\right) \\
& -\sum_{j=1}^{2} e_{i j}(t) \mu_{j}(t)-\bigwedge_{j=1}^{2} \alpha_{i j}(t) \\
& \cdot \int_{-\infty}^{t} K_{i j}(t-s) h_{j}\left(x_{j}(s)\right) d s \\
& -\bigwedge_{j=1}^{2} T_{i j}(t) \mu_{j}(t) \\
& -\bigvee_{j=1}^{2} \beta_{i j}(t) \int_{-\infty}^{t} K_{i j}(t-s) h_{j}\left(x_{j}(s)\right) d s \\
& \left.-\bigvee_{j=1}^{2} S_{i j}(t) \mu_{j}(t)-I_{i}(t)\right],
\end{aligned}
$$

where $i=1,2$ and

$$
\begin{gathered}
f_{1}(x)=f_{2}(x)=\frac{1}{2}(|x+1|+|x|-1), \\
g_{1}(x)=g_{2}(x)=\tan x, \\
h_{1}(x)=h(x)=\tanh x, \\
a_{1}\left(x_{1}(t)\right)=2-\sin \left(2 x_{1}(t)\right), \\
a_{2}\left(x_{2}(t)\right)=2-\cos \left(x_{1}(t)\right), \\
b_{1}\left(x_{1}(t)\right)=3 x_{1}(t), \\
b_{2}\left(x_{2}(t)\right)=4 x_{2}(t), \quad c_{11}(t)=0.05+0.01 \cos t, \\
c_{12}(t)=0.02+0.01 \sin t, \\
c_{21}(t)=0.02-0.01 \cos t, \\
c_{22}(t)=0.03+0.01 \sin t, \\
d_{11}(t)=0.06-0.01 \cos t, \\
d_{12}(t)=0.05-0.01 \sin t,
\end{gathered}
$$




$$
\begin{aligned}
& d_{21}(t)=0.05-0.02 \sin t, \\
& d_{22}(t)=0.07-0.02 \sin t \text {, } \\
& \alpha_{11}(t)=0.07+0.01 \cos t, \\
& \alpha_{12}(t)=0.03+0.02 \sin t \text {, } \\
& \alpha_{21}(t)=0.06+0.01 \sin t, \\
& \alpha_{22}(t)=0.06-0.02 \sin t \text {, } \\
& \beta_{11}(t)=0.01+0.03 \sin t, \\
& \beta_{12}(t)=0.05+0.03 \cos t, \\
& \beta_{21}(t)=0.03+0.04 \cos t, \\
& \beta_{22}(t)=0.01+0.04 \sin t, \\
& \tau_{i j}(t)=\sin ^{2} t, \quad K_{i j}(s)=e^{-s} . \\
& S_{11}(t)=0.23-0.22 \sin t, \\
& S_{12}(t)=0.27-0.02 \cos t, \\
& S_{21}(t)=0.03+0.45 \cos t, \\
& S_{22}(t)=0.14-0.07 \sin t, \\
& T_{11}(t)=0.15+0.24 \cos t, \\
& T_{12}(t)=0.17-0.03 \sin t, \\
& T_{21}(t)=0.14+0.03 \sin t, \\
& T_{22}(t)=0.15+0.07 \cos t, \\
& e_{11}(t)=0.03+0.02 \cos t \\
& e_{12}(t)=0.04+0.03 \sin t, \\
& e_{21}(t)=0.02+0.04 \sin t, \\
& e_{22}(t)=0.04-0.03 \cos t, \\
& \mu_{1}(t)=\sin t, \quad \mu_{2}(t)=\cos t, \\
& I_{1}(t)=-\cos ^{2}\left(e^{t}\right), \quad I_{2}(t)=-\sin \left(e^{t}\right) \text {. }
\end{aligned}
$$

By calculating, we have that $a_{1}^{-}=a_{2}^{-}=1, a_{1}^{+}=a_{2}^{+}=3, \lambda_{1}=3$, $\lambda_{2}=4, L_{j}^{f}=L_{j}^{g}=L_{j}^{h}=1, \int_{0}^{+\infty} K_{i j}(s) d s=1$, and $\tau=1$ and take the positive real numbers $\omega_{j}=1, j=1,2$; then

$$
\begin{aligned}
r=\max _{1 \leq i \leq n}\{ & \left(\lambda_{i} a_{i}^{-} \omega_{i}^{-1}\right)^{-1} \\
& {\left[\sum_{j=1}^{n} c_{i j}^{+} L_{j}^{f} a_{j}^{+} \omega_{j}+\sum_{j=1}^{n} d_{i j}^{+} L_{j}^{g} a_{j}^{+} \omega_{j}\right.} \\
& \left.\left.+\sum_{j=1}^{n}\left(\alpha_{i j}^{+}+\beta_{i j}^{+}\right) L_{j}^{h} a_{j}^{+} \omega_{j}\right]\right\}
\end{aligned}
$$

$$
=0.47<1
$$

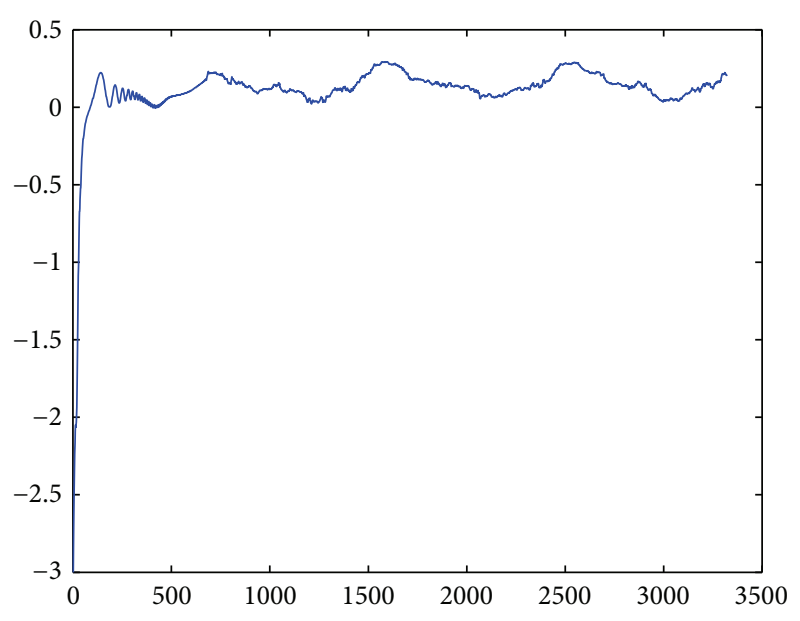

FIGURE 1: Behavior of the state component for $x_{1}(t)$.

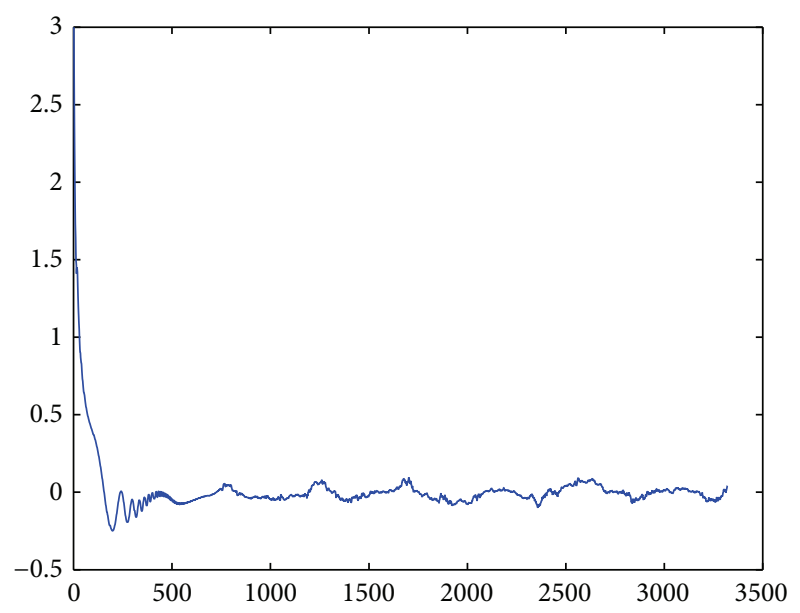

FIGURE 2: Behavior of the state component for $x_{2}(t)$.

Moreover, we have

$$
\begin{aligned}
& \sum_{j=1}^{2}\left(c_{1 j}^{+} L_{j}^{f} a_{j}^{+} \omega_{j}+d_{1 j}^{+} L_{j}^{g} a_{j}^{+} \omega_{j} e^{\mu \tau}\right. \\
& \left.\quad+\left(\alpha_{1 j}^{+}+\beta_{1 j}^{+}\right) L_{j}^{h} a_{j}^{+} \omega_{j} \int_{0}^{+\infty} K_{1 j}(s) e^{\mu s} d s\right) \\
& =1.41<3=\lambda_{1} a_{1}^{-} \omega_{1}, \\
& \sum_{j=1}^{2}\left(c_{2 j}^{+} L_{j}^{f} a_{j}^{+} \omega_{j}+d_{2 j}^{+} L_{j}^{g} a_{j}^{+} \omega_{j} e^{\mu \tau}\right. \\
& \left.\quad+\left(\alpha_{2 j}^{+}+\beta_{2 j}^{+}\right) L_{j}^{h} a_{j}^{+} \omega_{j} \int_{0}^{+\infty} K_{2 j}(s) e^{\mu s} d s\right) \\
& =1.17<4=\lambda_{2} a_{2}^{-} \omega_{2} .
\end{aligned}
$$

All the assumptions in Theorems 12 and 13 are satisfied. We set the initial condition $x(t)=\left[\begin{array}{ll}-3 & 3\end{array}\right]^{T}$; the response of the state component is showed in Figures 1 and 2. Therefore, (61) has an 
almost automorphic solution, which is globally exponentially stable.

\section{Conflict of Interests}

The authors declare that there is no conflict of interests regarding the publication of this paper.

\section{Acknowledgments}

The authors are deeply grateful to the reviewers for their careful reading of this paper and helpful comments, which have been very useful for improving the quality of this paper. This work is supported by the National Natural Sciences Foundation of China under Grant no. 11361072.

\section{References}

[1] B. Liu and L. Huang, "Existence and exponential stability of almost periodic solutions for cellular neural networks with time-varying delays," Physics Letters A, vol. 341, no. 1-4, pp. 135144, 2005.

[2] E. Y1lmaz, "Almost periodic solutions of impulsive neural networks at non-prescribed moments of time," Neurocomputing, vol. 141, pp. 148-152, 2014.

[3] L. Peng and W. Wang, "Anti-periodic solutions for shunting inhibitory cellular neural networks with time-varying delays in leakage terms," Neurocomputing, vol. 111, pp. 27-33, 2013.

[4] G. Zhang, Y. Shen, Q. Yin, and J. Sun, "Global exponential periodicity and stability of a class of memristor-based recurrent neural networks with multiple delays," Information Sciences, vol. 232, pp. 386-396, 2013.

[5] Y. Q. Ke and C. F. Miao, "Stability and existence of periodic solutions in inertial BAM neural networks with time delay," Neural Computing and Applications, vol. 23, no. 3-4, pp. 10891099, 2013.

[6] M. A. Cohen and S. Grossberg, "Absolute stability of global pattern formation and parallel memory storage by competitive neural networks," IEEE Transactions on Systems, Man, and Cybernetics, vol. 13, no. 5, pp. 815-826, 1983.

[7] Y. K. Li, "Existence and stability of periodic solutions for CohenGrossberg neural networks with multiple delays," Chaos, Solitons and Fractals, vol. 20, no. 3, pp. 459-466, 2004.

[8] T. Huang, C. Li, and G. Chen, "Stability of Cohen-Grossberg neural networks with unbounded distributed delays," Chaos, Solitons \& Fractals, vol. 34, no. 3, pp. 992-996, 2007.

[9] Z. Chen and J. Ruan, "Global dynamic analysis of general Cohen-Grossberg neural networks with impulse," Chaos, Solitons and Fractals, vol. 32, no. 5, pp. 1830-1837, 2007.

[10] C. Bai, "Global exponential stability and existence of periodic solution of Cohen-Grossberg type neural networks with delays and impulses," Nonlinear Analysis: Real World Applications, vol. 9, no. 3, pp. 747-761, 2008.

[11] Z. Yuan, D. Hu, L. Huang, and G. Dong, "Existence and global exponential stability of periodic solution for Cohen-Grossberg neural networks with delays," Nonlinear Analysis. Real World Applications, vol. 7, no. 4, pp. 572-590, 2006.

[12] T. Li, A. Song, and S. Fei, "Further stability analysis on CohenGrossberg neural networks with time-varying and distributed delays," International Journal of Systems Science, vol. 42, no. 10, pp. 1639-1649, 2011.
[13] T. Yang and L.-B. Yang, "The global stability of fuzzy cellular neural network," IEEE Transactions on Circuits and Systems. I. Fundamental Theory and Applications, vol. 43, no. 10, pp. 880883, 1996.

[14] Q. Song and J. Cao, "Impulsive effects on stability of fuzzy Cohen-Grossberg neural networks with time-varying delays," IEEE Transactions on Systems, Man, and Cybernetics, Part B: Cybernetics, vol. 37, no. 3, pp. 733-741, 2007.

[15] S. Niu, H. Jiang, and Z. Teng, "Exponential stability and periodic solutions of FCNNs with variable coefficients and time-varying delays," Neurocomputing, vol. 71, no. 13-15, pp. 2929-2936, 2008.

[16] Y. H. Xia, Z. Yang, and M. Han, "Lag synchronization of unknown chaotic delayed yang-yang-type fuzzy neural networks with noise perturbation based on adaptive control and parameter identification," IEEE Transactions on Neural Networks, vol. 20, no. 7, pp. 1165-1180, 2009.

[17] P. Balasubramaniam and M. S. Ali, "Robust exponential stability of uncertain fuzzy Cohen-Grossberg neural networks with time-varying delays," Fuzzy Sets and Systems, vol. 161, no. 4, pp. 608-618, 2010.

[18] G. Bao, S. P. Wen, and Z. G. Zeng, "Robust stability analysis of interval fuzzy Cohen-Grossberg neural networks with piecewise constant argument of generalized type," Neural Networks, vol. 33, pp. 32-41, 2012.

[19] R. Sakthivel, A. Arunkumar, K. Mathiyalagan, and S. M. Anthoni, "Robust passivity analysis of fuzzy Cohen-Grossbert BAM neural networks with time-varying delays," Applied Mathematics and Computation, vol. 218, no. 7, pp. 3799-3809, 2011.

[20] K. Mathiyalagan, J. H. Park, R. Sakthivel, and S. M. Anthoni, "Delay fractioning approach to robust exponential stability of fuzzy Cohen-Grossberg neural networks," Applied Mathematics and Computation, vol. 230, pp. 451-463, 2014.

[21] R. Sathy and P. Balasubramaniam, "Stability analysis of fuzzy Markovian jumping Cohen-Grossberg BAM neural networks with mixed time-varying delays," Communications in Nonlinear Science and Numerical Simulation, vol. 16, no. 4, pp. 2054-2064, 2011.

[22] C. Z. Li, Y. K. Li, and Y. Ye, "Exponential stability of fuzzy Cohen-Grossberg neural networks with time delays and impulsive effects," Communications in Nonlinear Science and Numerical Simulation, vol. 15, no. 11, pp. 3599-3606, 2010.

[23] S. Bochner, "Uniform convergence of monotone sequences of functions," Proceedings of the National Academy of Sciences of the United States of America, vol. 47, pp. 582-585, 1961.

[24] S. Bochner, "A new approach to almost periodicity," Proceedings of the National Academy of Sciences of the United States of America, vol. 48, pp. 2039-2043, 1962.

[25] S. Bochner, "Continuous mappings of almost automorphic and almost periodic functions," Proceedings of the National Academy of Sciences of the United States of America, vol. 52, pp. 907-910, 1964.

[26] G. M. N'Guerekata, Topics in Almost Automorphy, Springer, New York, NY, USA, 2005.

[27] T. Diagana, Almost Automorphic Type and Almost Periodic Type Functions in Abstract Spaces, Springer, Berlin, Germany, 2013.

[28] G. M. N’Guerekata, "Existence and uniqueness of almost automorphic mild solutions to some semilinear abstract differential equations," Semigroup Forum, vol. 69, no. 1, pp. 80-86, 2004.

[29] J. A. Goldstein and G. M. N'Guerekata, "Almost automorphic solutions of semilinear evolution equations," Proceedings of the American Mathematical Society, vol. 133, no. 8, pp. 2401-2408, 2005. 
[30] K. Ezzinbi and G. M. N’Guérékata, "Almost automorphic solutions for some partial functional differential equations," Journal of Mathematical Analysis and Applications, vol. 328, no. 1, pp. 344-358, 2007.

[31] F. Cherif and Z. B. Nahia, "Global attractivity and existence of weighted pseudo almost automorphic solution for GHNNs with delays and variable coefficients," Gulf Journal of Mathematics, vol. 1, pp. 5-24, 2013.

[32] F. Chérif, "Sufficient conditions for global stability and existence of almost automorphic solution of a class of RNNs," Differential Equations and Dynamical Systems, vol. 22, no. 2, pp. 191-207, 2014.

[33] Y. Li and L. Yang, "Almost automorphic solution for neutral type high-order Hopfield neural networks with delays in leakage terms on time scales," Applied Mathematics and Computation, vol. 242, pp. 679-693, 2014.

[34] J. H. Sun and L. Wan, "Global exponential stability and periodic solutions of Cohen-Grossberg neural networks with continuously distributed delays," Physica D: Nonlinear Phenomena, vol. 208, no. 1-2, pp. 1-20, 2005.

[35] Y. K. Li, X. R. Chen, and L. Zhao, "Stability and existence of periodic solutions to delayed Cohen-Grossberg BAM neural networks with impulses on time scales," Neurocomputing, vol. 72, no. 7-9, pp. 1621-1630, 2009.

[36] Y. K. Li and X. L. Fan, "Existence and globally exponential stability of almost periodic solution for Cohen-Grossberg BAM neural networks with variable coefficients," Applied Mathematical Modelling: Simulation and Computation for Engineering and Environmental Systems, vol. 33, no. 4, pp. 2114-2120, 2009.

[37] Z. Chen, D. H. Zhao, and J. Ruan, "Almost periodic attractor for Cohen-Grossberg neural networks with delay," Physics Letters A, vol. 373, no. 4, pp. 434-440, 2009.

[38] H. J. Xiang, J. H. Wang, and J. D. Cao, "Almost periodic solution to Cohen-Grossberg-type BAM networks with distributed delays," Neurocomputing, vol. 72, no. 16-18, pp. 3751-3759, 2009.

[39] Y. Li, T. Zhang, and Z. Xing, "The existence of nonzero almost periodic solution for Cohen-Grossberg neural networks with continuously distributed delays and impulses," Neurocomputing, vol. 73, no. 16-18, pp. 3105-3113, 2010.

[40] H. J. Xiang and J. D. Cao, "Almost periodic solution of CohenGrossberg neural networks with bounded and unbounded delays," Nonlinear Analysis: Real World Applications, vol. 10, no. 4, pp. 2407-2419, 2009.

[41] W. G. Yang, "Periodic solution for fuzzy Cohen-Grossberg BAM Neural networks with Both Time-Varying and distributed delays and variable coefficients," Neural Processing Letters, vol. 40, pp. 51-73, 2014. 


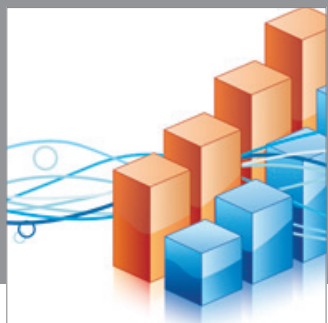

Advances in

Operations Research

mansans

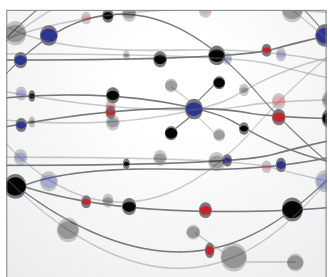

The Scientific World Journal
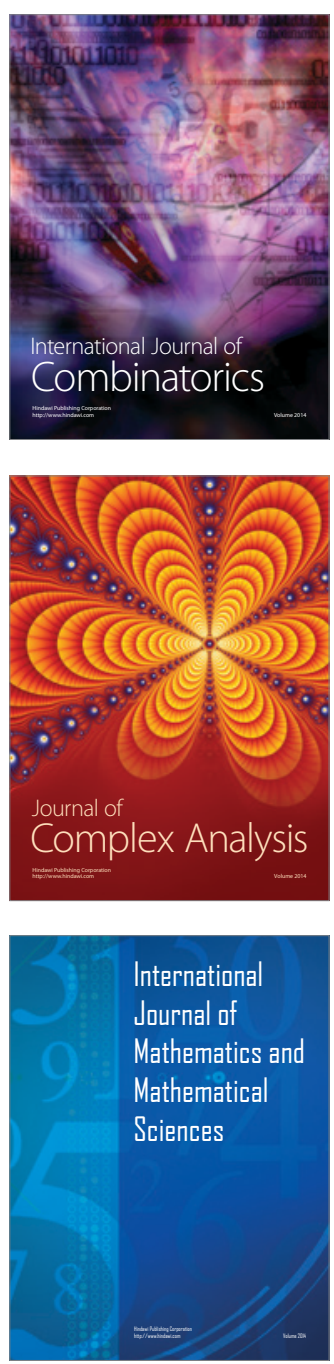
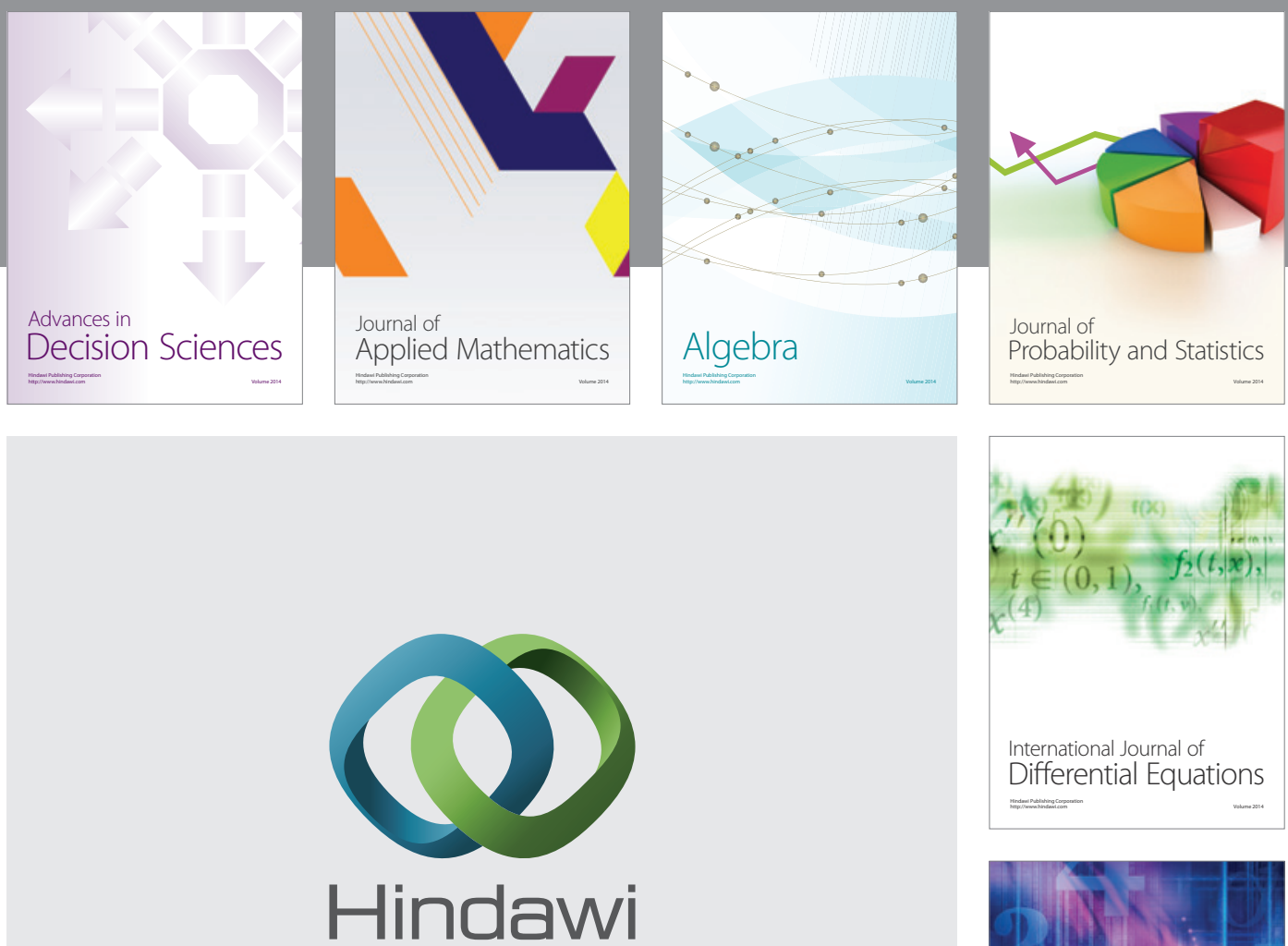

Submit your manuscripts at http://www.hindawi.com
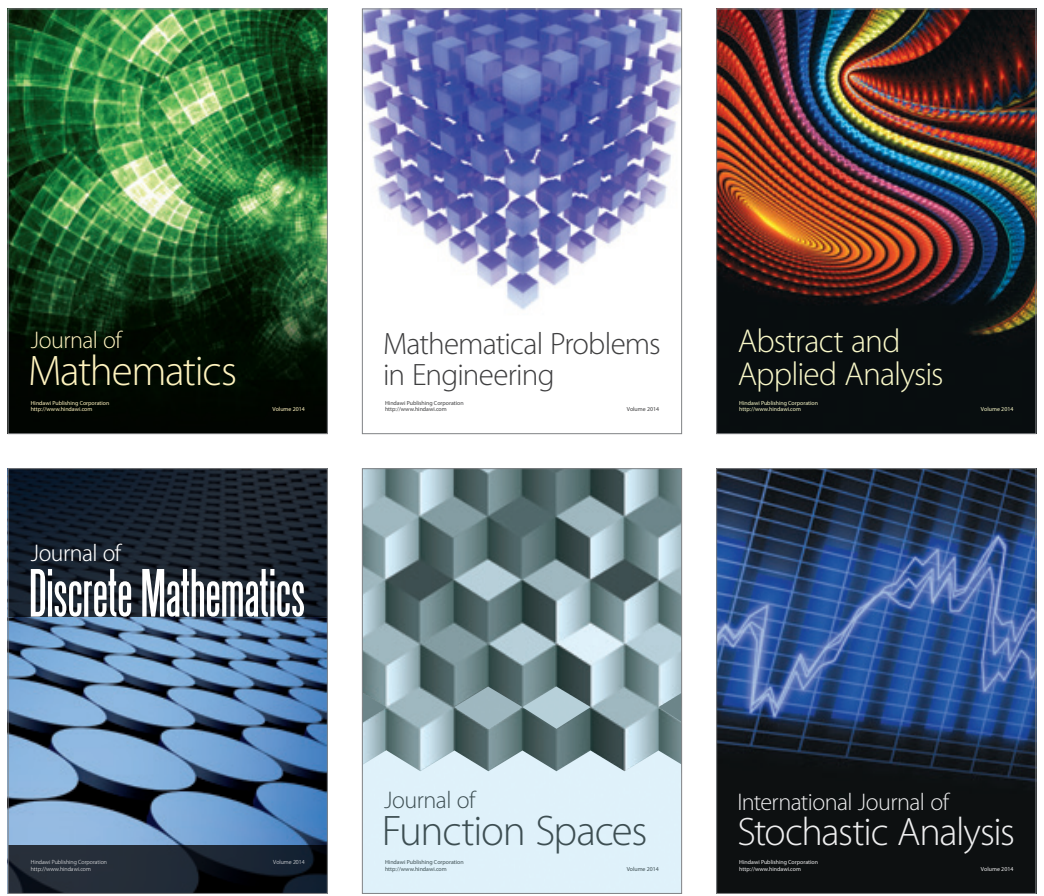

Journal of

Function Spaces

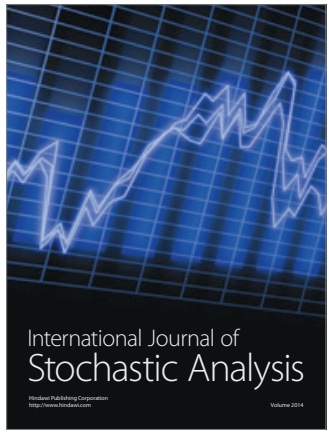

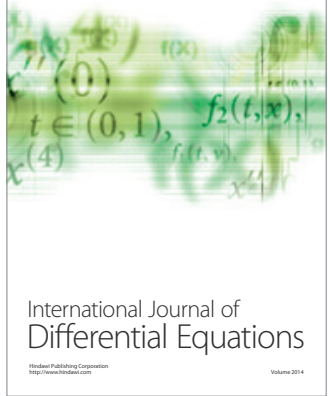
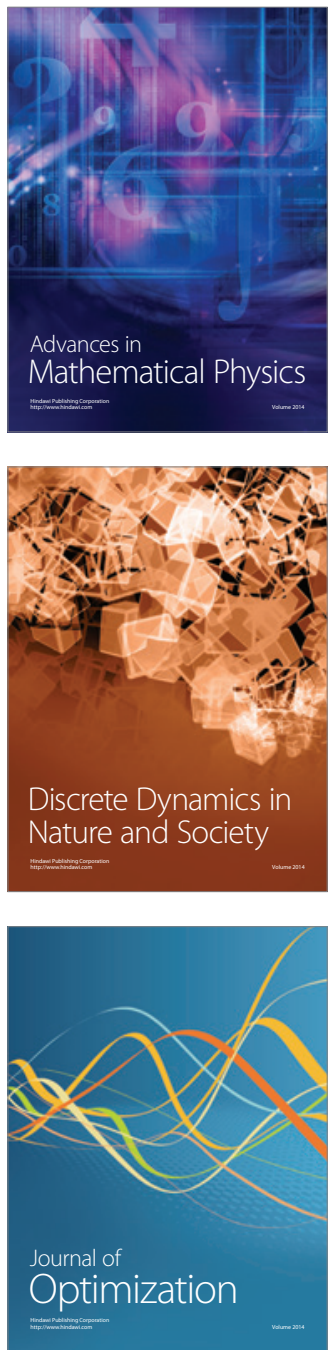